\title{
BMJ Open Flash glucose monitoring in gestational diabetes mellitus: study protocol for a randomised controlled trial
}

\author{
Agata Majewska (D) , Paweł Stanirowski, Mirosław Wielgoś, Dorota Bomba-Opoń
}

To cite: Majewska A, Stanirowski P, Wielgoś M, et al. Flash glucose monitoring in gestational diabetes mellitus: study protocol for a randomised controlled trial. BMJ Open 2021;11:e041486. doi:10.1136/ bmjopen-2020-041486

- Prepublication history and supplemental material for this paper is available online. To view these files, please visit the journal online (http://dx.doi. org/10.1136/bmjopen-2020041486).

Received 09 June 2020 Revised 20 January 2021 Accepted 25 January 2021

Check for updates

(C) Author(s) (or their employer(s)) 2021. Re-use permitted under CC BY-NC. No commercial re-use. See rights and permissions. Published by BMJ.

1st Department of Obstetrics and Gynecology, Medical University of Warsaw, Warsaw, Poland

Correspondence to Dorota Bomba-0poń; dorota.bomba-opon@wum. edu.pl

\section{ABSTRACT}

Introduction Gestational diabetes mellitus (GDM) is a glucose intolerance occurring in $3 \%-10 \%$ of pregnant women and being a risk factor for multiple maternal and fetal complications. The risk of perinatal complications is proportional to the level of maternal hyperglycaemia. Proper glycaemic control is therefore one of the key elements of GDM therapy. Until recently, determination of blood glucose concentration was performed using glucose meters, which involved multiple fingerpricks. Nowadays, due to the flash glucose monitoring (FGM) availability, it is possible to collect measurements at any time without routine puncturing. The aim of the presented study is to assess the impact of FGM on the efficacy of treatment in population of patients diagnosed with GDM.

Methods and analysis This is a prospective, randomised study, that will recruit 100 women at 24-28 weeks of gestation at the 1st Department of Obstetrics and Gynecology, Medical University of Warsaw, Poland. Women diagnosed with GDM, who will meet the inclusion criteria, will be individually randomised to the FGM or self-monitoring of blood glucose groups. Further on, clinical and laboratory results of the mother and their newborns will be collected for analysis during the course of pregnancy. Primary outcome is mean glycaemia result in each group after 1 month analysis and percentage of results in the target glycaemic range. The secondary objectives will be to compare the two groups for maternal and neonatal outcomes in conjunction with long-term glycaemic control using blood glycated haemoglobin and fructosamine serum concentrations.

Ethics and dissemination The study is exempt from regional ethics review due to its nature of quality improvement in patient care. The study has been approved by the Bioethics Committee at the Medical University of Warsaw and the patient privacy protection boards governing over the recruitment sites. Results of the study will be presented in peer-reviewed journals and at conferences.

Trial registration number NCT04422821.

\section{INTRODUCTION}

Gestational diabetes mellitus (GDM) is a glucose intolerance diagnosed for the first time in pregnancy. It affects 3\%-10\% of pregnant women and is a risk factor for multiple maternal and fetal complications. ${ }^{1}$ During pregnancy, GDM significantly increases the
Strengths and limitations of this study

- Flash Glucose Monitoring In Gestational Diabetes Mellitus (FLAMINGO) Study is a prospective, crossover, open-label and randomised trial.

- FLAMINGO Study will compare flash glucose monitoring with self-monitoring of blood glucose in glycaemia control in gestational diabetes mellituscomplicated pregnancies.

- FLAMINGO trial protocol adopts rigorous methodology and is written in accordance with the Standard Protocol Items: Recommendations for Interventional Trials.

- Flash glucose monitoring device will be applied only for the one-third of the trial due to budgetary restrictions.

- The trial will include small groups of participants at a single obstetric department, that is a limitation of the study.

risk of fetal macrosomia, shoulder dystocia, birth trauma and caesarean section. ${ }^{2}$ Furthermore, the long-term complications of GDM include increased risk of development of diabetes mellitus type 2 in the mother, ${ }^{3}$ as well as increased risk of obesity, diabetes and metabolic syndrome occurrence in their children. ${ }^{3}{ }^{4}$ It has been well documented that the risk of above-mentioned complications increases with the level of maternal hyperglycaemia. ${ }^{5}$ Proper glycaemic control is one of the key elements in the effective treatment of GDM. Until recently, glucose monitoring was solely performed using glucose meters, which required multiple fingerpricks (self-monitoring of blood glucose, SMBG). Nowadays, due to the glycaemia monitoring systems development, such as flash glucose monitoring (FGM), glucose levels may be measured less invasively through subcutaneous sensor application. FGM is a factory-calibrated sensor measuring glucose concentrations in the interstitial fluid. Although it represents different measurement technique than SMBG, previous studies have shown that glycaemia levels obtained 
by both methods are comparable. ${ }^{6}$ Apart from continuous glucose concentration measurements, the system provides additional data by creating a 24-hour glycaemic profile. In comparison, SMBG provides only single, intermittent measurements, that limit detection of glycaemic variability or nocturnal hypoglycaemic events. ${ }^{6}$ As shown in one of the studies, FGM due to the ease of use was three times more often applied as a method of glycaemia control than SMBG. As a result, patients from FGM group had significantly better blood glucose control. ${ }^{7}$ In the study by Bühling et al, continuous glucose monitoring had overall better sensitivity in detecting abnormal glucose levels. ${ }^{8}$ Furthermore, results of the IMPACT Study (Novel glucose-sensing technology and hypoglycaemia in type 1 diabetes: a multicentre, non-masked, randomised controlled trial) demonstrated that the use of FGM in patients with diabetes mellitus type 1 significantly reduced the incidence of hypoglycaemia episodes. ${ }^{7}$ Although hyperglycaemia is the most common alteration occurring in GDM patients, there is also an increased risk of masked hypoglycaemia. It was showed that almost one third of GDM patients experienced hypoglycaemic events during the course pregnancy that could have been easily detected using methods for continuous glycaemia control. ${ }^{9}$ Diagnosis of these hypoglycaemic episodes may be of particular importance in GDM patients before qualifying to insulin therapy.

The main purpose of our study is to evaluate the impact of a new method of glycaemia control (FGM) on the efficacy of treatment of GDM. Assuming that the first month after diagnosis of GDM is essential for the proper implementation of dietary and physical activity recommendations by the patients, the favourable cost-effective strategy will be to apply FGM device only during that period of pregnancy. By analysing results of this study, such as fasting and postprandial glycaemia levels, number of nocturnal hypoglycaemic episodes, number of women requiring insulin therapy, daily dosage of insulin and maternalfetal perinatal outcomes, we will provide a scientific basis for more common use of FGM in the population of pregnant women affected by GDM.

\section{METHODS AND ANALYSIS}

The study protocol was written in accordance with the Standard Protocol Items: Recommendations for Interventional Trials statement.

\section{Study design}

FLAMINGO (Flash Glucose Monitoring In Gestational Diabetes Mellitus) trial is a single-centre, non-blinded, randomised, cross-over study with a nested qualitative evaluation and 1:1 allocation ratio. The study will be conducted at the 1st Department of Obstetrics and Gynecology, Medical University of Warsaw, Poland over a period of 2020-2021.

\section{Study population and eligibility criteria}

We aim to recruit 100 pregnant women diagnosed with GDM based on the results of $75 \mathrm{~g}$ oral glucose tolerance test, performed between 24 and 28 gestational weeks, in accordance with the universal criteria defined by WHO. ${ }^{10}$ Patients will be randomly divided into two groups: FGM-study group comprising 50 women who will receive subcutaneous sensor for glucose monitoring, and SMBG-control group comprising 50 women who will monitor glycaemia through use of standard glucose meter.

\section{Inclusion criteria}

Women aged 18 years or older, in singleton pregnancy, diagnosed with GDM will be invited to participate in the study.

\section{Exclusion criteria}

Multiple pregnancy, fetal malformations, PGDM (overt diabetes in pregnancy), chronic or pregnancy-induced hypertension, chronic renal or hepatic disease diagnosed prior to study entry, in vitro fertilisation, premature rupture of membranes, placenta previa, smoking in pregnancy, intake of medications including: methyldopa, tetracyclin, acetylosalicylic acid, acetaminofen, ibuprofen, L-dopa, tolazamide, tolbutamide will constitute study exclusion criteria.

All women eligible for the study will provide written informed consent prior to enrollment.

\section{Aim of the study and objectives}

The aim of the FLAMINGO trial is to assess the impact of FGM on the efficacy of treatment of GDM. Our primary outcome is mean glycaemia results (fasting and 1 hour postprandial glucose concentrations) in each group (FGM/SMBG) during the first month following GDM diagnosis in conjuction with the percentage of results in the target glycaemic range. The secondary objectives will be to compare the two groups for the number of patients requiring insulin therapy, dosage of insulin, number of hypoglycaemic episodes, as well as to compare blood glycated haemoglobin (HbAlc) and fructosamine serum concentrations as potential markers of long-term glycaemic control and predictors of perinatal complications, based on previous studies. ${ }^{11} 12$ Simultaneously, we aim to compare patient's physical activity level based on a daily steps number counted by a wristband and to assess compliance with diet recommendations using Eating Assessment Test. Finally, we aim to compare both groups with respect to maternal-fetal perinatal outcomes, including pregnancy weight gain, fetal birth weight and neonatal glycaemia.

\section{Participant selection and recruitment}

Pregnant women diagnosed with GDM by the trial research staff (AM, PS) who will meet study inclusion criteria will be invited to participate in the project (see figure 1). Recruitment will begin in September 2020 and is estimated to end in October 2021.

Recruitment brochures that contain general information of the study will be placed at the website of the Department. During the recruitment process, trial research staff 
Potential Participants: Inclusion

criteria $\rightarrow 18$ years old; GDM

diagnosed between 24-28 weeks' gestation
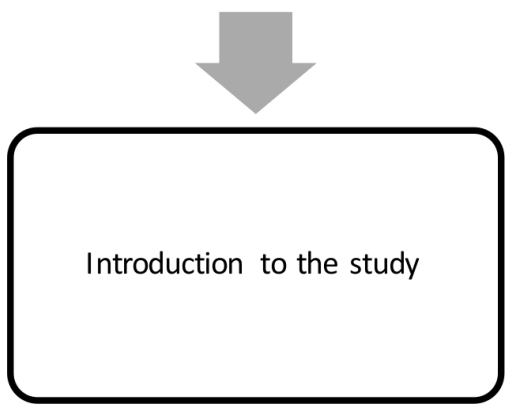

Accept to participate

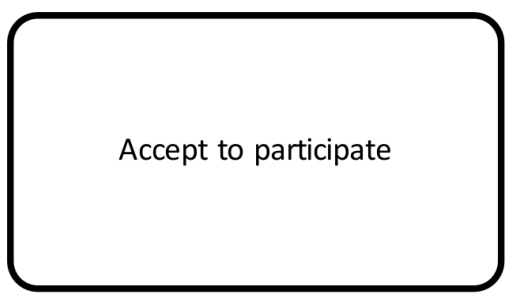

Evaluation: Exclusion criteria

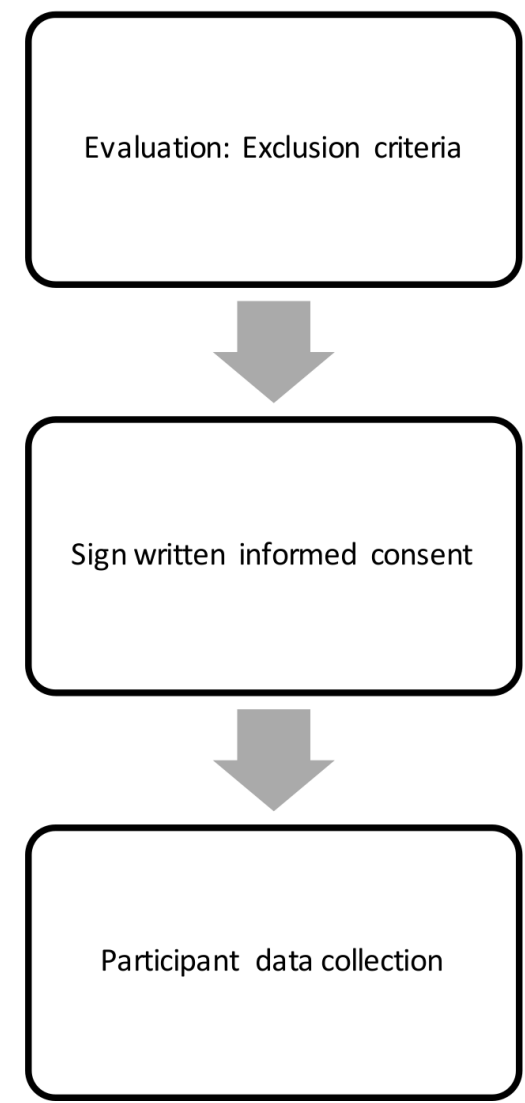

Figure 1 Patient flow scheme. GDM, gestational diabetes mellitus.
(AM, PS) will inform potential candidates about the study both verbally and with written information. Women who are agreeable to participate will be obliged to provide written informed consent. Those patients who decline to participate will continue to receive their routine antenatal care. Obstetric care provided to each pregnant woman will not be affected nor influenced by the woman's decision to either participate or not participate in the study.

\section{Randomisation}

Simple randomisation with the computer-generated list and sealed envelopes will be used for patient's randomisation process. The process of randomisation and sealing will be conducted at the 1st Department of Obstetrics and Gynecology, Medical University of Warsaw by the nonmember of the trial research staff.

\section{Blinding}

This is a non-blinded trial. As the device used for glycaemia monitoring (FGM/SMBG) will be clearly visible to both participants and trial research staff blinding is not feasible for patients and researchers.

\section{Study procedures}

Patients included in the study will undergo five visits comprising one recruitment and four follow-up visits (see figure 2). The trial research staff (AM, PS) will be responsible for analysing participants glycaemia results, diet control and physical activity as well as for the modifications of health interventions during the follow-up visits.

\section{Recruitment visit (visit 1)}

At the recruitment visit (24-28 weeks of gestation), after providing the informed consent, patients will be interviewed for sociodemographic data and medical history to analyse study exclusion criteria. Next, if eligible for the study, participants will be randomly divided into two consecutive groups. Simple randomisation with the computer-generated list and sealed envelopes will be used for patient's randomisation process. Study group (FGM) will comprise 50 women who will receive subcutaneous sensor for glucose monitoring (FreeStyle Libre; Abbott Diabetes Care, Alameda, California, USA), and the control group (SMBG) will comprise 50 women who will monitor glycaemia through use of standard glucose meter (iXell; Genexo sp; Warsaw, Poland; ISO 15197:2015). All patients from the study group will obtain instruction for using Freestyle Libre app to measure and collect glycaemia results using a mobile phone. Patients without mobile phone will obtain Freestyle Libre Reader and instructions for using the device. Accordingly, control group will be informed about proper use of glucose meters. The results from FGM and SMBG will be collected during the follow-up visits.

In order to assess daily physical activity, all participants will obtain a wristband (Xiaomi Mi Band 4; Xiaomi Corporation, Hong Kong) allowing for footsteps measurement. According to Polish Society of Obstetricians and Gynecologists (PSOG), the recommended number of footsteps in 


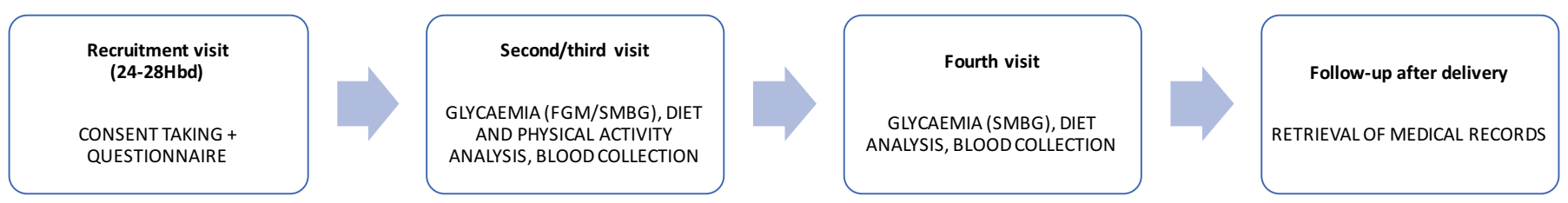

Figure 2 The flow diagram of the study. FGM, flash glucose monitoring; SMBG, self-monitoring of blood glucose.

pregnancy is 10000 per day. ${ }^{13}$ As previously demonstrated, mild physical activity, such as walking has protective effect on excessive gestational weight gain and decreases the risk of preterm birth and fetal macrosomia. ${ }^{14-17}$

All patients included in the study will obtain the instruction for using the wristband and its mobile app, together with recommendations about daily physical activity in pregnancy.

After meeting, study inclusion criteria patient will obtain diet recommendations for GDM prepared by clinical dietician. To evaluate participants' dietary habits, we will use Eating Assessment Test prepared by the Polish National Institute of Public Health-National Institute of Hygiene. This is a short questionnaire (20 items for diet) intended to evaluate dietary habits in patients diagnosed with GDM. The summary points base on a number of meals per day, length of breaks between meals, daily portions of fruits and vegetables, frequency of fried food and sweets consumption per week (questionnaire and list of points for each element provided in online supplemental file). The maximum test result is 42 points, the minimum 4 points. Based on the points obtained, patients will be assigned to one of the four diet groups (good: 39-42, satisfactory: 30-38, demanding diet modification: $12-29$ and not satisfactory: $<12$ points).

According to PSOG recommendations, all participants will be obliged to measure fasting and 1 hour postprandial glucose concentrations in a daily manner, together with once per week midnight measurement. ${ }^{18}$ Postprandial glucose measurements will be performed after three main meals (breakfast, dinner, supper). To avoid excessive data collection, we do not aim to analyse all the results obtained with the FGM. At the end of the visit, all participants will undergo blood tests (HbAlc, fructosamine) and selected biometric maternal-fetal parameters will be measured (patient weight, ultrasound estimated fetal weight).

\section{Visit 2 (14 days after the recruitment)}

At the second follow-up visit, glycaemia levels, diet control and physical activity will be analysed. Based on glycaemia results, participants will obtain modified diet recommendations and will be qualified to insulin therapy, if required (fasting blood glucose concentrations above $90 \mathrm{mg} / \mathrm{dL}$ or postprandial glycaemia results above $140 \mathrm{mg} / \mathrm{dL}$ ). Initial predetermined insulin dose planned for the study is four units for long-acting insulin and three units per meal for short-acting insulin. The final insulin dosage will be individualised based on the glycaemic results during the follow-up visits. ${ }^{19}$ According to PSOG standards for management of GDM, fasting glucose level $<90 \mathrm{mg} /$ $\mathrm{dL}$ and 1 hour postprandial glucose level $<140 \mathrm{mg} / \mathrm{dL}$ are indicative of proper glycaemic control. ${ }^{18}$ The study group will obtain new FGM sensor. At the end of the visit, research staff will collect biometric measurements from all patients included in the study (the same as during Visit $1)$.

According to the criteria proposed by Tudor-Locke, we will divide patients into four groups of physical activity based on a number of daily steps collected by the wristband: sedentary (<5000 daily steps), low active (50007500 daily steps), somewhat active (7500-10000 daily steps) and active ( $\geq 10000$ daily steps) ${ }^{20}$

\section{Visit 3 (28 days after the recruitment)}

At the third visit, glycaemia levels, diet control and physical activity will be analysed. Based on glycaemia results, participants will obtain modified diet recommendations and will be qualified to insulin therapy if required. In patients already treated with insulin, dosage will be modified according to glycaemia results. The study group will obtain glucose meter. All patients will end physical activity control with a wristband. Eating Assessment Test will be performed to test whether dietary recommendations and glycaemia control in the study have an impact on eating habits of the participants. At the end of the visit, all participants will undergo blood tests (HbAlc, fructosamine) and selected biometric parameters will be measured.

\section{Visit 4 (34-36 gestational weeks)}

At the fourth visit, glycaemia levels, diet control and insulin therapy will be analysed. Based on glycaemia results, participants will obtain modified diet recommendations and will be qualified to insulin therapy, if required. In patients already treated with insulin, dosage will be modified according to glycaemia results. Eating Assessment Test will be performed to test whether dietary recommendations and glycaemia control in the study have an impact on eating habits of the participants. Additionally, research staff will collect selected biometric measurements from all patients included in the study. At 
the end of the visit, all participants will undergo blood tests (HbA1c, fructosamine).

\section{Visit 5 (after delivery)}

After the delivery (24-72hours), research staff will retrieve maternal and neonatal outcomes from patient's medical history.

\section{Study outcome}

The primary outcome will be mean glycaemia results (fasting and 1 hour postprandial glucose concentrations) in each group (FGM/SMBG) during the first month following the diagnosis of GDM and the percentage of results in the target glycaemic range. The secondary objectives will be to compare both groups for:

- Number of patients requiring insulin therapy (2, 4 and 8 weeks after the recruitment visit).

- Dosage of insulin (2, 4 and 8 weeks after the recruitment visit).

- Long-term glycaemic control using blood HbAlc concentrations $(2,4$ and 8 weeks after the recruitment visit).

- Long-term glycaemic control using fructosamine serum concentrations $(2,4$ and 8 weeks after the recruitment visit).

- Number of hypoglycaemic episodes (glucose concentration $<70 \mathrm{mg} / \mathrm{dL}$ ) during 1 month analysis (episodes per day in 0-4 weeks after the recruitment visit).

- Physical activity during 1 month analysis based on a footsteps daily count $(0-4$ weeks after the recruitment visit).

- Compliance with diet recommendations according to eating assessment test (2, 4 and 8 weeks after the recruitment visit).

- Gestational weight gain (2, 4 and 8 weeks after the recruitment visit).

- Mode of delivery (rate of vaginal delivery/ caesarean section).

- Fetal birth weight.

- Neonatal glycaemia.

\section{Sample size calculation and statistical analysis}

The performed power analysis (power of $80 \%$, significance level of $5 \%$, two-sided) estimated a required sample size of a total of 80 patients (40 patients in each group). The analysis was based on the results of a previous report comparing FGM with SMBG and estimation to detect a difference in the percentage of results in the target glycaemic range between study and control groups. ${ }^{21}$ Sample size is further increased to 100 patients to account for a potential exclusions and drop out of approximately $10 \%$.

Continuous data will be compared using the MannWhitney $\mathrm{U}$ test, and for categorical variables the $\chi^{2}$ test will be applied. The results will be presented as medians and IQRs or as a frequency (\%). For comparison between groups, Bland Altman and Passing-Bablok method will be performed. The relationship between glucose, HbAlc and fructosamine concentrations, and selected maternal-fetal parameters will be examined with the use of Pearson's correlation coefficient. Multivariable logistic regression analysis will be performed to evaluate the potential impact of selected predictors on primary outcomes. All tests will be carried out at a significance level of 0.05 .

Statistical analyses will be performed using SAS software, V.9.2 or later (SAS Institute).

\section{Patient and public involvement}

Patients and the manufacturers (Abbott Diabetes Care, Genexo sp, Xiaomi Corporation) will not be involved in the process of the design, conduct, reporting or dissemination plans of the study. All participants will be informed about the trial results at the end of the study.

\section{Trial monitoring and management}

\section{Patient retention strategy}

Patients recruited to the study will obtain an email with the schedule of the follow-up visits. They will also obtain regular phone contact before each visit.

\section{Strategies for the management of missing data}

For missing glycaemic data, in particular in the SMBG group mean glycaemia levels from the past 5 days will be calculated, in accordance with the time of measurement (fasting, postprandial).

\section{ETHICS AND DISSEMINATION}

The study is exempt from regional ethics review due to its nature of quality improvement in patient care. The study has been approved by the Bioethics Committee at the Medical University of Warsaw and the patient privacy protection boards governing over the recruitment sites. All data sets will be password protected and only available to project investigators. Results of the study will be presented in peer-reviewed journals and at conferences.

\section{DISCUSSION}

According to epidemiological data, GDM affects about $5.4 \%$ of pregnant women in Europe and the prevalence is continuously increasing. ${ }^{22}$ As a result, there is an urgent need to search for the new methods of effective glucose monitoring facilitating glycaemia control, and thus allowing for a decrease in the rate of maternal and fetal complications.

FGM is a new method, that is already commonly used in paediatric patients diagnosed with diabetes mellitus type $1 .^{23}$ As one multicentre study demonstrated, due to easiness of FGM use, patients measured glycaemia three times more often per day in comparison with those using standard glucose meters. Consequently, patients significantly improved their diurnal glucose profile. ${ }^{7}$ In addition, the results of IMPACT Study proved that use of FGM among type 1 diabetic patients is effective in reduction of 
hypoglycaemic episodes. ${ }^{23}$ Finally, in the study by Dunn et $\mathrm{al}$, a positive correlation between the number of scans per day and HbA1c levels was found, showing a significant decrease in HbA1c concentration among patients who monitored glycaemia more frequently. ${ }^{24}$

Apart from the relatively small group of participants, the presented single-centre study is limited by the fact that FGM will be applied only for 1 month following the GDM diagnosis. Nonetheless, we believe that these first 4 weeks after the recruitment are of crucial importance for proper implementation of dietary and physical activity recommendations by the patients. ${ }^{25}$ Moreover, if primary objectives are accomplished proposed strategy may significantly reduce costs of FGM application.

To conclude, although limited in number, available studies suggest that use of FGM may help to improve monitoring and treatment results in patients affected by glucose tolerance disorders during pregnancy. Based on that, we would like to investigate the impact of FGM on maternal and neonatal outcomes in population of patients diagnosed with GDM.

Contributors $\mathrm{AM}$ is the principal investigator and coordinated trial design, drafting and critically revising of the protocol. PS and DB-O were involved in drafting the manuscript for publication. AM and PS developed the statistical design and sample size estimation. PS, DB-0 and MW critically revised and supervised the trial design and protocol. DB-0 and MW contributed to the ethical and regulatory aspects of the research. All the authors have read and approved the final version of the manuscript.

Funding This study (including sensors, glucose meters and wristbands) will be funded by the 1st Department of Obstetrics and Gynecology, Medical University of Warsaw. The manufacturers (Abbott Diabetes Care, Genexo sp, Xiaomi Corporation) have no role in the process of the design, funding, conducting, reporting, dissemination plans of the study, data management and publication.

Competing interests None declared.

Patient consent for publication Not required.

Ethics approval The Bioethics Committee at the Medical University of Warsaw; Approval Number: KB/50/2020

Provenance and peer review Not commissioned; externally peer reviewed.

Supplemental material This content has been supplied by the author(s). It has not been vetted by BMJ Publishing Group Limited (BMJ) and may not have been peer-reviewed. Any opinions or recommendations discussed are solely those of the author(s) and are not endorsed by BMJ. BMJ disclaims all liability and responsibility arising from any reliance placed on the content. Where the content includes any translated material, BMJ does not warrant the accuracy and reliability of the translations (including but not limited to local regulations, clinical guidelines, terminology, drug names and drug dosages), and is not responsible for any error and/or omissions arising from translation and adaptation or otherwise.

Open access This is an open access article distributed in accordance with the Creative Commons Attribution Non Commercial (CC BY-NC 4.0) license, which permits others to distribute, remix, adapt, build upon this work non-commercially, and license their derivative works on different terms, provided the original work is properly cited, appropriate credit is given, any changes made indicated, and the use is non-commercial. See: http://creativecommons.org/licenses/by-nc/4.0/.

ORCID iD

Agata Majewska http://orcid.org/0000-0001-8071-0368

\section{REFERENCES}

1 Wan CS, Abell S, Aroni R, et al. Ethnic differences in prevalence, risk factors, and perinatal outcomes of gestational diabetes mellitus: a comparison between immigrant ethnic Chinese women and Australian-born Caucasian women in Australia. J Diabetes 2019:11:809-17.

2 Mirghani Dirar A, Doupis J. Gestational diabetes from a to Z. World J Diabetes 2017:8:489-511.

3 Damm P, Houshmand-Oeregaard A, Kelstrup L, et al. Gestational diabetes mellitus and long-term consequences for mother and offspring: a view from Denmark. Diabetologia 2016;59:1396-9.

4 Dugas C, Perron J, Kearney M, et al. Postnatal prevention of childhood obesity in offspring prenatally exposed to gestational diabetes mellitus: where are we now? Obes Facts 2017;10:396-406.

5 Scott EM, Bilous RW, Kautzky-Willer A. Accuracy, user acceptability, and safety evaluation for the FreeStyle Libre flash glucose monitoring system when used by pregnant women with diabetes. Diabetes Technol Ther 2018;20:180-8.

6 Bailey T, Bode BW, Christiansen MP, et al. The performance and usability of a Factory-Calibrated flash glucose monitoring system. Diabetes Technol Ther 2015;17:787-94.

7 Bolinder J, Antuna R, Geelhoed-Duijvestijn P, et al. Novel glucosesensing technology and hypoglycaemia in type 1 diabetes: a multicentre, non-masked, randomised controlled trial. Lancet 2016;388:2254-63.

8 Bühling KJ, Kurzidim B, Wolf C, et al. Introductory experience with the continuous glucose monitoring system (CGMS; medtronic Minimed) in detecting hyperglycemia by comparing the selfmonitoring of blood glucose (SMBG) in non-pregnant women and in pregnant women with impaired glucose tolerance and gestational diabetes. Exp Clin Endocrinol Diabetes 2004;112:556-60.

9 Naik D, Hesarghatta Shyamasunder A, Doddabelavangala Mruthyunjaya $\mathrm{M}$, et al. Masked hypoglycemia in pregnancy. $J$ Diabetes 2017;9:778-86.

10 Diagnostic criteria and classification of hyperglycaemia first detected in pregnancy: a world Health organization guideline. Diabetes Res Clin Pract 2014;103:341-63.

11 Ayyappan S, Philips S, Kumar CK, et al. Serum fructosamine a better indicator than glycated hemoglobin for monitoring gestational diabetes mellitus. J Pharm Bioallied Sci 2015;7:S32-4.

12 Mendes N, Alves M, Andrade R, et al. Association between glycated albumin, fructosamine, and $\mathrm{HbA} 1 \mathrm{c}$ with neonatal outcomes in a prospective cohort of women with gestational diabetes mellitus. Int $J$ Gynaecol Obstet 2019;146:326-32.

13 Wender-Ozegowska E, Bomba-Opoń D, Brazert J, et al. [Recommendations of Polish Gynecological Society concerning perinatal care in obese pregnant women]. Ginekol Pol 2012;83:795-9.

14 Takito MY, Benício Maria Helena D'Aquino, Benicio MH. Physical activity during pregnancy and fetal outcomes: a case-control study. Rev Saude Publica 2010;44:90-101.

15 Jiang H, Qian X, Li M, et al. Can physical activity reduce excessive gestational weight gain? findings from a Chinese urban pregnant women cohort study. Int J Behav Nutr Phys Act 2012;9:12.

16 Connolly CP, Conger SA, Montoye AHK, et al. Walking for health during pregnancy: a literature review and considerations for future research. J Sport Health Sci 2019;8:401-11.

17 Marshall MR, Montoye AHK, George AJ. Pregnancy walking cadence does not vary by trimester. Gait Posture 2018;65:81-5

18 Wender-Ożegowska E, Bomba-Opoń D, Brązert J, et al. Ginekologicznego: Postępowanie U kobiet Z cukrzycą. Ginekol. Pol 2017;2:215-29.

19 American Diabetes Association. Insulin administration. Diabetes Care 2003;26 Suppl 1:S121-4.

20 Tudor-Locke C, Bassett DR. How many steps/day are enough? preliminary pedometer indices for public health. Sports Med 2004;34:1-8.

21 Costa D, Lourenço J, Monteiro AM, et al. Clinical performance of flash glucose monitoring system in patients with liver cirrhosis and diabetes mellitus. Sci Rep 2020;10:7460.

22 Eades CE, Cameron DM, Evans JMM. Prevalence of gestational diabetes mellitus in Europe: a meta-analysis. Diabetes Res Clin Pract 2017:129:173-81.

23 Mancini G, Berioli MG, Santi E, et al. Flash glucose monitoring: a review of the literature with a special focus on type 1 diabetes. Nutrients 2018;10. doi:10.3390/nu10080992. [Epub ahead of print: $29 \mathrm{Jul} 2018]$

24 Dunn TC, Xu Y, Hayter G, et al. Real-World flash glucose monitoring patterns and associations between self-monitoring frequency and glycaemic measures: a European analysis of over 60 million glucose tests. Diabetes Res Clin Pract 2018:137:37-46.

25 Campbell FM, Murphy NP, Stewart C, et al. Outcomes of using flash glucose monitoring technology by children and young people with type 1 diabetes in a single arm study. Pediatr Diabetes 2018;19:1294-301. 